\title{
CONHECIMENTO DE ENFERMEIROS SOBRE A EXECUÇÃO E INTERPRETAÇÃO DO ECG: UMA REVISÃO INTEGRATIVA
}

\author{
KNOWLEDGE OF NURSES ON ECG EXECUTION AND INTERPRETATION: AN \\ INTEGRATIVE REVIEW
}

\section{RESUMO}

Identificar à luz da literatura, estudos que avaliaram o conhecimento de enfermeiros sobre a execução e interpretação do ECG. Trata-se de uma revisão integrativa da literatura que utilizou como fonte de pesquisa as bases eletrônicas e bibliotecas virtuais: Literatura Latino-Americana e do Caribe em Ciências da Saúde, Base de Dados de Enfermagem, Biblioteca Virtual Scientific Eletronic Library Online e Medical Literature Analysis and Retrieval System Online no período de 2008 a abril de 2018, utilizando os descritores "Enfermagem", "Eletrocardiografia" e "Cuidados críticos". Foram encontrados 43 artigos que após a aplicação dos critérios de inclusão e exclusão oito atenderam os objetivos do estudo. Identificou-se as seguintes categorias: Atuação do enfermeiro na execução do ECG em pacientes com dor torácica, Habilidade, conhecimento teórico-prático e interpretação do ECG entre os profissionais de enfermagem. Infere-se que uma prática de educação permanente em serviço sobre a eletrocardiografia e a concretização de estudos de intervenções poderá contribuir com melhorias para realização e interpretação do procedimento bem como estudos com maior nível de evidência com vistas a corroborar com a conscientização e a importância do procedimento.

Palavras-chave: Enfermagem. Eletrocardiografia. Cuidados Críticos.

\section{ABSTRACT}

To identify, in the light of the literature, studies that evaluated nurses' knowledge about the performance and interpretation of the electrocardiogram. This is an

1 Graduada em Enfermagem pelo Centro Universitário de João Pessoa (UNIPÊ). E-mail: aede_silva@hotmail.com 2 Doutoranda em Nutrição pela Universidade Federal da Paraíba (UFPB). Docente do Centro Universitário de João Pessoa (UNIPÊ). E-mail: keyth.sulamitta.lima@gmail.com

3 Mestre em Enfermagem pela Universidade de Pernambuco (UPE). Docente do Centro Universitário de João Pessoa (UNIPÊ). E-mail: prof_carlos_narciso@yahoo.com.br

4 Mestre em Enfermagem pela Universidade Federal da Paraíba (UFPB). Docente do Centro Universitário de João Pessoa (UNIPÊ). E-mail: ronnyufpb@gmail.com 
integrative review of the literature that used as a source of research the electronic databases and virtual libraries: Latin American and Caribbean Literature in Health Sciences, Nursing Database, Virtual Library Scientific Electronic Library Online and Medical Literature Analysis and Retrieval System Online from 2008 to April 2018, using the descriptors "Nursing", "Electrocardiography" and "Critical Care". 43 articles were found that, after applying the inclusion and exclusion criteria, eight met the study objectives. The following categories were identified: Nurse performance in ECG execution in patients with chest pain, Ability, theoretical-practical knowledge and ECG interpretation among nursing professionals. It is inferred that a practice of permanent education in service on electrocardiography and the accomplishment of intervention studies may contribute with improvements to the performance and interpretation of the procedure as well as studies with a higher level of evidence to corroborate with the awareness and the importance of the procedure.

Keywords: Nursing. Electrocardiography. Critical Care.

\section{INTRODUÇÃO}

O Eletrocardiograma (ECG) é um gráfico que representa a atividade elétrica do coração. O traçado eletrocardiográfico apresenta uma série de ondas, intervalos, complexos e segmentos, em que é possível identificar patologias através da captação dos impulsos elétricos do músculo cardíaco como o Infarto Agudo do Miocárdio (IAM) bem como as arritmias (BARROS, 2016).

As doenças cardiovasculares causam cerca de $70 \%$ de todos os óbitos no mundo, totalizando em média 38 milhões de óbitos anuais. Dessas mortes, 16 milhões ocorrem em pessoas com idade inferior a 70 anos e aproximadamente 28 milhões, em países com renda baixa e média (MALTA et al., 2017). O IAM é registrado como a primeira causa de óbitos no País, e anualmente são apontados através da base de dados do Departamento de Informática do SUS (DATASUS), cerca de 100 mil óbitos devido à doença.

O ECG é um exame simples que auxilia no processo de diagnóstico do IAM, anormalidades de condução, compartimentos cardíacos dilatados, disritmias, e níveis baixos ou elevados de cálcio e potássio (GONZALEZ et al., 2013; BRASIL, 2014).

Na interpretação do ECG, é necessário que o enfermeiro tenha um nível de conhecimento baseado na fundamentação teórica de anatomia e fisiologia humana, fisiopatologias e patologias cardiológicas, evidências clínicas e áreas afins (SOUZA; LIMA, 2013).

O profissional de enfermagem precisa ter habilidade para identificar possíveis alterações no ECG, analisando em conjunto a clínica do paciente para poder intervir dentro do contexto de suas práticas. Para isso, faz-se necessária atualização e capacitação para poder realizar um bom trabalho junto com 
sua equipe, buscando sempre atuar nas situações que são consideradas como inesperadas na realidade que está inserido (ALVES et al., 2013)

A capacitação do enfermeiro para execução do ECG é indispensável para reconhecer as necessidades de pacientes cardíacos, a fim de prestar um cuidado sistêmico e humanizado, qualificado, individualizado e sistematizado. Ele é um dos profissionais da equipe assistencial que permanece consecutivamente ao lado do paciente, portanto é de extrema importância que seja capaz de realizar a interpretação do ECG, identificando traçados eletrocardiográficos normais e patológicos para que com isso possa intervir de modo adequado e imediato (LOPES; FERREIRA, 2013).

Um recente ensaio clínico randomizado feito 134 estudantes do terceiro ano do curso de graduação em enfermagem da Universidade de Atenas, avaliou duas estratégias de ensino para interpretação das arritmias cardíacas e concluiu que todos os estudantes apresentaram dificuldade para reconhecer as taquiarritmias (SANTANA-SANTOS et al., 2017). Nesse sentido justifica-se esse estudo por ser pertinente aprofundar o conhecimento teórico e prático na execução e interpretação do ECG, com o intuito de reduzir e minimizar riscos e complicações as quais poderão ser identificadas a tempo de realizar uma intervenção adequada e assim foi utilizada a seguinte questão norteadora: qual o conhecimento dos enfermeiros acerca do ECG apontados na literatura científica de Enfermagem?

Assim, objetivo desse estudo é verificar a luz da literatura científica em enfermagem o conhecimento de enfermeiros acerca do ECG.

\section{METODOLOGIA}

Trata-se de uma revisão integrativa da literatura, dividida em seis etapas: Elaboração da questão norteadora; definição dos critérios de inclusão e exclusão; seleção dos estudos que irão compor a amostra; organização e categorização dos estudos; análise e interpretação dos resultados; e relato da revisão que sintetizam estudos anteriores sobre um determinado tema, analisando informações já produzidas e conhecimentos sobre questões que podem ser respondidas com novos estudos (MENDES; SILVEIRA; GALVÃO, 2008).

A fim de embasar o levantamento dos dados, foi utilizada a seguinte questão norteadora: quais são as evidências disponíveis, na literatura, sobre o conhecimento dos enfermeiros acerca do ECG?

Como critérios de inclusão foram adotados: estudos que tinham como linha principal da pesquisa a realização e interpretação do eletrocardiograma pelo enfermeiro, os que se encontravam disponíveis na íntegra, gratuitamente, em português e espanhol, e por fim aqueles publicados entre 2008 e 2018. Foram excluídos os estudos que estavam em duplicidade na mesma ou em outra base de dados e aqueles que não abordavam a temática como eixo central. 
Os dados foram coletados entre os meses de março e abril de 2018, por meio de consulta nas seguintes bases eletrônicas: Literatura Latino-Americana e do Caribe em Ciências da Saúde (LILACS), Base de Dados de Enfermagem (BDENF), Biblioteca Virtual Scientific Eletronic Library Online (SciELO) e na Medical Literature Analysis and Retrieval System Online (MEDLINE). O termo utilizado para a busca selecionado a partir dos Descritores em Ciências da Saúde (DeCS) foram "Enfermagem" , "Eletrocardiografia", e "Cuidados Críticos" combinados por meio do conector booleano "AND".

Após a leitura dos títulos e resumos houve a aplicação dos critérios de inclusão e exclusão procedendo-se com a leitura dos artigos selecionados na íntegra. Na etapa seguinte com a finalidade de organizar as informações foi construída uma planilha através do software Microsoft Office Excel $2016 \mathrm{com}$ as variáveis: título, base de dados, periódico, tipo de estudo e nível de evidência, ano de publicação e país.

Os artigos foram elegidos e classificados através do nível de evidência, e para esta revisão foi utilizado um sistema de classificação dividido em sete níveis, sendo: Nível I - evidências oriundas de revisões sistemáticas ou meta-análise de relevantes ensaios clínicos; Nível II - evidências derivadas de pelo menos um ensaio clínico randomizado controlado bem delineado; Nível III - ensaios clínicos bem delineados sem randomização; Nível IV - estudos de coorte e de caso controle bem delineados; Nível $\vee$ - revisão sistemática de estudos descritivos e qualitativos; Nível VI - evidências derivadas de um único estudo descritivo ou qualitativo e Nível VII opinião de autoridades ou relatório de comitês de especialistas (STILLWELL, 2010).

Os autores referenciados foram devidamente citados, respeitando e identificando as fontes de pesquisa, com extrema observância no tocante ao rigor ético quanto à propriedade intelectual dos textos que foram analisados.

\section{RESULTADOS}

Inicialmente foram encontrados 43 estudos, que após a aplicação dos critérios de inclusão e exclusão restaram oito produções que abrangem o corpus da pesquisa. Dentre eles estavam presentes quatro na LILACS, quatro na BDENF e nenhum na biblioteca virtual SciELO e MEDLINE. De acordo com a figura abaixo (Figura 1). 
Figura 1 - Diagrama de fluxo para seleção dos estudos, João Pessoa, Paraíba, Brasil, 2018.

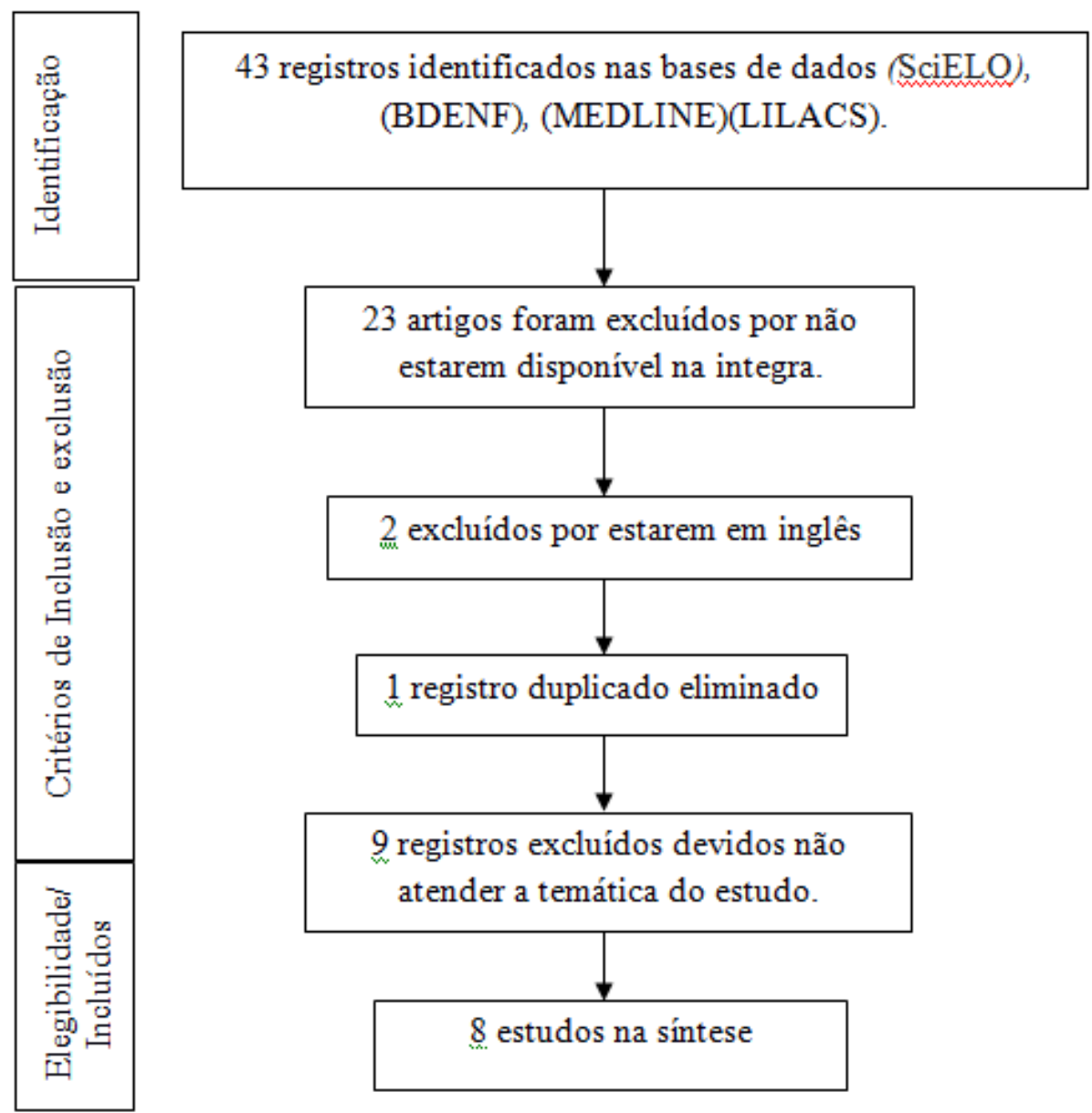

Na LILACS foram encontradas 36 publicações que após a leitura de títulos e resumos, foram excluídas 21 por não estarem disponíveis na íntegra, nove por não estarem inseridos na temática, dois por se encontrarem em outro idioma, permanecendo quatro artigos (50\%) disponíveis para amostra final.

$\mathrm{Na}$ BDENF sete publicações foram encontradas, duas foram excluídas por não se encontrarem disponíveis na íntegra e uma duplicada restando quatro artigos (50\%) para amostra final.

No tocante aos periódicos houve uma maior produção na Revista Baiana de enfermagem com dois artigos (28\%), seguido da Revista Gaúcha de Enfermagem (12\%), da Revista Enfermagem Centro Oeste Mineiro (12\%), Ateneu (12\%), Revista enfermagem UFPE on line (12\%), International Journal of Cardiovascular Sciences: IJCS (12\%), Revista Enfermagem Instituto Mexicano Seguro Social (12\%).

Em relação ao tipo de estudo, observou-se um quantitativo maior do tipo transversal com quatro estudos (48\%) sendo correlacional, quantitativos e descritivos, um estudo do tipo exploratório com abordagem descritiva e quantitativa (13\%), um 
estudo correlacional descritivo (13\%), um estudo observacional retrospectivo (13\%), e um estudo de coorte (13\%).

Em relação aos níveis de evidência houve uma maior produção de estudos com nível de evidência VI contabilizando sete estudos (96\%), um com nível IV (4\%). No que tange ao ano de publicação houve uma maior produção nos anos de 2015 com três (60\%), seguido por 2017 com dois (25\%), 2009, 2014 e 2018 com um cada (15\%).

Por fim, pertinente ao país, a produção maior esteve presente no Brasil com seis artigos (70\%), subsequente ao Peru com um (15\%) e, finalmente o México com um (15\%).

Com o intuito de facilitar a compreensão dos resultados evidenciados, as buscas incluídas nesta revisão ficaram agrupadas por categorias: Título, base de dados/biblioteca, periódico, tipo de estudo/nível de evidência, autores, ano e país, conforme evidenciado no Quadro 1.

Quadro 1 - Sinopse dos estudos elencados para compor a revisão integrativa da literatura. João Pessoa, Paraíba, Brasil, 2018.

\begin{tabular}{|c|c|c|c|c|c|c|}
\hline TíTULO & $\begin{array}{l}\text { BASE DE } \\
\text { DADOS/ } \\
\text { BIBLIOTECA }\end{array}$ & PERIÓDICO & $\begin{array}{c}\text { TIPO DE } \\
\text { ESTUDO/NÍVEL } \\
\text { DE EVIDÊNCIA }\end{array}$ & AUTORES & $\begin{array}{l}\mathrm{A} \\
\mathbf{N} \\
\mathrm{O}\end{array}$ & $\begin{array}{l}\text { P } \\
\text { A } \\
\text { I } \\
\text { s }\end{array}$ \\
\hline $\begin{array}{c}\text { Tempoporta- } \\
\text { eletrocardiograma } \\
\text { (ECG): } \\
\text { Um indicador } \\
\text { de eficácia no } \\
\text { tratamento do infarto } \\
\text { agudo do miocárdio }\end{array}$ & LILACS & $\begin{array}{c}\text { Revista } \\
\text { Gaúcha } \\
\text { Enfermagem }\end{array}$ & $\begin{array}{l}\text { Estudo de } \\
\text { coorte, IV }\end{array}$ & $\begin{array}{l}\text { SOARES,T.; } \\
\text { SOUZA E.N.; } \\
\text { MORAES M.A.; } \\
\text { AZZOLINK. }\end{array}$ & 2009 & BRASIL \\
\hline $\begin{array}{c}\text { Dor torácica: } \\
\text { Atuação do } \\
\text { enfermeiro em um } \\
\text { pronto atendimento } \\
\text { de um hospital escola }\end{array}$ & BDENF & $\begin{array}{c}\text { Revista } \\
\text { Enfermagem } \\
\text { Centro Oeste } \\
\text { Mineiro }\end{array}$ & $\begin{array}{l}\text { Estudo do tipo } \\
\text { exploratório, } \\
\text { com uma } \\
\text { abordagem } \\
\text { descritiva e } \\
\text { quantitativa, VI }\end{array}$ & $\begin{array}{l}\text { CAVEIÃO C, } \\
\text { SANTOS,R.B.; } \\
\text { MONTEZELI J.H } \\
\text { et al. }\end{array}$ & 2014 & BRASIL \\
\hline $\begin{array}{c}\text { Conocimientos y } \\
\text { calidad del registro } \\
\text { en enfermeras sobre } \\
\text { electrocardiograma } \\
\text { en el servicio de } \\
\text { emergencia del } \\
\text { Hospital Guillermo } \\
\text { Almenara Irigoyen }\end{array}$ & LILACS & Ateneo & $\begin{array}{c}\text { Estudo } \\
\text { quantitativo, } \\
\text { método } \\
\text { descritivo } \\
\text { de corte } \\
\text { transversal, VI. }\end{array}$ & $\begin{array}{l}\text { COLLANTES } \\
\text { MEJÍA, M. G. }\end{array}$ & 2015 & PERÚ \\
\hline $\begin{array}{l}\text { Conhecimento } \\
\text { teórico-prático de } \\
\text { enfermeiras sobre o } \\
\text { eletrocardiograma }\end{array}$ & LILACS & $\begin{array}{c}\text { Revista } \\
\text { Baiana de } \\
\text { Enfermagem }\end{array}$ & $\begin{array}{c}\text { Pesquisa } \\
\text { transversal, VI }\end{array}$ & $\begin{array}{l}\text { FERNANDES, L. } \\
\text { S.; LIRA, M. C. L. } \\
\text { S.; FRANÇA,V. } \\
\text { V.; VALOIS,A. A.; } \\
\text { VALENÇA, M. P. }\end{array}$ & 2015 & BRASIL \\
\hline
\end{tabular}




\begin{tabular}{|c|c|c|c|c|c|c|}
\hline $\begin{array}{l}\text { Avaliação do } \\
\text { sistema de triagem } \\
\text { de Manchester na } \\
\text { síndrome coronariana } \\
\text { aguda / Evaluation of } \\
\text { the Manchester triage } \\
\text { system in the acute } \\
\text { coronary sydrome }\end{array}$ & LILACS & $\begin{array}{l}\text { International } \\
\text { Journal of } \\
\text { Cardiovascular } \\
\text { Sciences: IJCS }\end{array}$ & $\begin{array}{l}\text { Estudo } \\
\text { observacional } \\
\text { retrospectivo, } \\
\text { VI }\end{array}$ & $\begin{array}{l}\text { GOUVÊA, V. E. } \\
\text { T.; REIS, M. A. } \\
\text { M.; GOUVEA, } \\
\text { G. M.; LIMA, H. } \\
\text { N.; ABUARA, A. }\end{array}$ & 2015 & BRASIL \\
\hline $\begin{array}{l}\text { Habilidade dos } \\
\text { enfermeiros na } \\
\text { interpretação do } \\
\text { eletrocardiograma de } \\
12 \text { derivações }\end{array}$ & BDENF & $\begin{array}{l}\text { Revista Baiana } \\
\text { Enfermagem }\end{array}$ & $\begin{array}{c}\text { Estudo } \\
\text { transversal, VI }\end{array}$ & $\begin{array}{l}\text { SANTOS,E. S.; } \\
\text { PIRES, E. C.: } \\
\text { SILVA, J. T et al. }\end{array}$ & 2017 & BRASIL \\
\hline $\begin{array}{c}\text { Correlação de dados } \\
\text { clínicos, laboratoriais } \\
\text { e eletrocardiográficos } \\
\text { com lesões obstrutivas } \\
\text { coronariana na } \\
\text { síndrome coronariana } \\
\text { aguda }\end{array}$ & BDENF & $\begin{array}{c}\text { Revista } \\
\text { enfermagem } \\
\text { UFPE on line }\end{array}$ & $\begin{array}{c}\text { Estudo } \\
\text { transversal e } \\
\text { correlacional } \\
\text { de abordagem } \\
\text { quantitativa, VI }\end{array}$ & $\begin{array}{l}\text { SANTOS,V.B.; } \\
\text { LOPES J. L.; } \\
\text { LOPES, C. Tet al. }\end{array}$ & 2017 & BRASIL \\
\hline $\begin{array}{l}\text { Competencia clínica } \\
\text { y conocimiento } \\
\text { teórico-práctico del } \\
\text { personal de } \\
\text { enfermería sobre } \\
\text { electrocardiografía } \\
\text { en pacientes adultos }\end{array}$ & BDENF & $\begin{array}{c}\text { Revista } \\
\text { Enfermagem } \\
\text { Instuto } \\
\text { Mexicano } \\
\text { Seguro Social }\end{array}$ & $\begin{array}{l}\text { Estudo } \\
\text { correlacional } \\
\text { descritivo, VI }\end{array}$ & $\begin{array}{l}\text { ALCANTAR, C. E. } \\
\text { S.; ESPARZA, C. } \\
\text { M.; CARDONA, } \\
\text { J.P. G. }\end{array}$ & 2018 & MÉXICO \\
\hline
\end{tabular}

Fonte: Elaborado pelo autor, João Pessoa, Paraíba, Brasil, 2018.

Posteriormente a leitura atenta aos estudos foram distribuídos em duas categorias para discussão: Atuação do enfermeiro na execução do ECG em pacientes com dor torácica; Habilidade, conhecimento teórico-prático e interpretação do ECG entre os profissionais de enfermagem.

\section{DISCUSSÃO}

\section{ATUAÇÃO DO ENFERMEIRO NA EXECUÇÃO DO ECG EM PACIENTES COM DOR TORÁCICA}

Os estudos selecionados para essa categoria identificaram que na unidade de urgência e emergência, a atuação do enfermeiro diante do paciente com dor torácica deve ser de ação imediata e a realização do ECG em pelo menos dez minutos além da dosagem de enzimas cardíacas, com intuito de iniciar o mais breve possível a terapêutica trombolítica, quando necessário. No entanto, para isso, os enfermeiros da unidade necessitam estar preparados para um atendimento de qualidade, estabelecendo uma comunicação adequada entre toda equipe (SOARES et al., 2009). 
Em um estudo realizado por Caveião et al. (2014) após entrevistar nove enfermeiros, conclui que cinco afirmam que caracterizam como principal sintoma a precordialgia e a irradiação para mandíbula, seis fazem a monitorização cardíaca e oito solicitam o ECG, fazendo-se necessário aplicação de um protocolo ou rotina para padronização do atendimento e diagnóstico precoce ao paciente com IAM. Complementa que a assistência de enfermagem deverá reconhecer seus sinais e sintomas a tempo de intervir, realizando os procedimentos emergenciais adequados. Portanto, faz toda diferença a atuação do enfermeiro no momento do atendimento ao paciente de urgência, levando em consideração seu papel enquanto membro da equipe multidisciplinar durante a atuação no protocolo de dor torácica bem como a autonomia para realização das condutas de enfermagem e do ECG.

De acordo com Santos et al. (2017) em seu estudo transversal e correlacional de abordagem quantitativa através de 160 prontuários de pacientes adultos com dor torácica secundário a síndrome coronariana aguda (SCA), as alterações mais comuns encontradas no ECG são: necrose miocárdica; distúrbios de condução intraventricular (bloqueios de ramos) e anormalidades no ritmo cardíaco, isquemia miocárdica; corrente de lesão. Os biomarcadores cardíacos indicados para o diagnóstico e manuseio de pacientes com SCA são mioglobina, creatinofosfoquinase (CK) e sua fração Mb e a troponina sendo evidenciado a injúria miocárdica quando ocorre elevação na concentração sérica. As principais atividades realizadas pelo enfermeiro na triagem dos pacientes com dor precordial incluem a avaliação da história clínica, realização do ECG, coleta dos biomarcadores e o exame físico.

Gouvêa etal. (2015) em seu estudo aos prontuários de 191 pacientes com SCA, $65 \%$ homens e $47 \%$ com antecedentes familiares de doença arterial coronariana, afirmam que mesmo que haja classificação correta do enfermeiro no serviço de urgência, um dos fatores agravantes para o prognóstico do paciente, é a demora para buscar um atendimento emergencial, além do mais, ao chegar ao prontoatendimento, a demora pra ser atendido também torna-se mais um agravante.

\section{HABILIDADE, CONHECIMENTO TEÓRICO-PRÁTICO E INTERPRETAÇÃO DO ECG ENTRE OS PROFISSIONAIS DE ENFERMAGEM}

Santana-Santos et al. (2017) afirmam em sua pesquisa que os enfermeiros que lidam com pacientes em estado críticos, tem mais habilidade e segurança para executar o ECG do que aqueles enfermeiros que não atuam e ainda ressaltam a importância de treinamento de toda equipe para prestação de uma assistência de qualidade.

Corroborando com este problema e em busca de uma solução viável, recomenda-se que os profissionais de enfermagem ao se formarem, busquem se especializar e realizem cursos de aperfeiçoamento, para que possa dessa forma contribuir com uma melhor assistência e reduzir agravos aos pacientes. 
Nesse contexto, Fernandes et al. (2015) destacam em seu estudo com 47 enfermeiras da Unidade de Terapia Intensiva e Cardiologia que nenhuma das enfermeiras responderam adequadamente o questionário sobre a técnica de localização e posicionamento dos eletrodos precordiais, tendo em vista a necessidade de treinamento em serviço e a importância de intervenções educativas, tais como educação permanente na unidades de urgências com a intenção de prestar um atendimento eficaz e de qualidade através dos profissionais de enfermagem.

Collantes Mejía (2015) complementa chamando a atenção dos enfermeiros que sentem dificuldades ao realizar e interpretar o ECG para que reflitam a respeito da situação atual do seu trabalho e busquem meios de implementar programas de educação contínua e/ou permanente visando melhorar, atualizar e fortalecer a base teórica e práticas fundamentais em ECG, com o intuito de melhorarem sua função e competência profissional.

A relação entre o conhecimento teórico e prático sobre o ECG, pode melhorar com formação e grau acadêmico. Portanto, ao adquirir capacitação e treinamento, a equipe de enfermagem poderá obter o melhor diagnóstico, realizando planejamento, intervenção e avaliação dos pacientes (ALCANTAR, 2018).

\section{CONSIDERAÇÕES FINAIS}

Foi possível com este estudo, observar a necessidade de educação permanente e treinamento com a finalidade de contribuir na formação dos profissionais de enfermagem, tendo em vista a real necessidade do conhecimento teórico-prático para interpretação de exames importantes como o ECG, devendo demonstrar destreza, agilidade e habilidades específicas, com o intuito de manter assistência de forma rápida, consciente e segura ao paciente.

Entretanto, é necessário a realização de estudos de intervenções para diagnosticar in loco as dificuldades dos profissionais de enfermagem, frente a realização e diagnóstico prévio do ECG. Assim, esperamos sinalizar instituições e centros formadores da importância de promover o aprimoramento do ensino bem como da técnica para realização e interpretação do ECG pelos enfermeiros.

\section{REFERÊNCIAS}

ALCANTAR, C. E. S.; ESPARZA, C. M.; CARDONA, J. P. G. Competencia clínica y conocimiento teórico-prático del personal de enfermeira sobre electrocardiografia em pacientes adultos. Revista Enferm Instituto Mexicano Seguro Social. v. 26, n. 1, p. 29-33, 2018. 
ALVES, T.E. et al. Atuação do enfermeiro no atendimento emergencial aos usuários acometidos de infarto agudo do miocárdio. Rev enferm UFPE on line., Recife, v.7, n. 1, p. 176-83, jan. 2013.

BARROS, A.L.B.L. Anamnese e exame físico: avaliação diagnostica de enfermagem no adulto. 3.ed. Porto Alegre: Artmed, 2016.

BRASIL. Ministério da Saúde. Portal da Saúde. Departamento de Informática do SUS - 2014. Disponível em: <http://datasus.saude.gov.br/noticias/atualizacoes/559infarto-agudo-do-miocardio-e-primeira-causa-de-mortes-no-pais-revela-dados-dodatasus>. Acesso em: 03 set 2017.

CAVEIÃO, C. et al. Dor torácica: Atuação do enfermeiro em um pronto atendimento de um hospital escola. Revista Enfermagem Centro Oeste Mineiro, v. 4, n.1, p. 921 928, 2014.

COLLANTES MEJÍA, M. G. Conocimientos y calidad del registro en enfermeras sobre electrocardiograma en el servicio de emergencia del Hospital Guillermo Almenara Irigoyen. Trabajo de Investigación (Especialista en Enfermería en Emergencias y Desastres). Lima, Perú: Universidad Nacional Mayor de San Marcos, Facultad de Medicina Humana, Escuela de Post-Grado, 2015.

FERNANDES, L. S. et al. Conhecimento teórico-prático de enfermeiras sobre o eletrocardiograma. Revista Baiana de Enfermagem, v. 29, n. 2, p. 98-105, abr/jun. 2015 .

GONZALEZ, M. M. et al. I Diretriz de Ressuscitação Cardiopulmonar e Cuidados Cardiovasculares de Emergência da Sociedade Brasileira de Cardiologia. Arq Bras Cardiol. v. 101, n. 2, p. 1-221, 2013.

GOUVÊA, V. E. T. et al. Avaliação do Sistema de Triagem de Manchester na Síndrome Coronariana Aguda. Internacional Journal of Cardiovascular Sciences. v. 28, n. 2, p. 107-113, 2015.

LOPES J.L.; FERREIRA, F. G. Eletrocardiograma para enfermeiros. São Paulo: Atheneu; 2013.

MALTA, D. C. et al. Doenças crônicas não transmissíveis e a utilização de serviços de saúde: análise da Pesquisa Nacional de Saúde no Brasil. Rev Saude Publica, v. 51, Supl p.1-4s, 2017.

MENDES, K. D. S.; SILVEIRA, R. C. C. P.; GALVAO, C. M. Revisão integrativa: método de pesquisa para a incorporação de evidências na saúde e na enfermagem. Texto contexto - enferm. [online]. vol.17, n.4, p.758-764, 2008. 
SANTANA-SANTOS, E. et al. Habilidade dos enfermeiros na interpretação do eletrocardiograma de 12 derivações. Revista Baiana Enfermagem., v. 31, n. 1 p. 165 81, 2017.

SANTOS, V. B. et al. Correlação de dados clínicos, laboratoriais e eletrocardiográficos com lesões obstrutivas coronariana na síndrome coronariana aguda. Revista enfermagem UFPE online. Recife, v. 11, n. 1, p. 319-26, 2017.

SOARES, T. et al. Tempo porta-eletrocardiograma (ECG): um indicador de eficáciano tratamento do infarto agudo do miocárdio. Rev Gaúcha Enferm., Porto Alegre (RS), v. 30, n. 1, p. 120-6, 2009.

SOUZA, L. P.; LIMA, M. G. Atuação do enfermeiro na realização e interpretação do eletrocardiograma (ECG) em unidade de terapia Intensiva (UTI) Revista UNINGÁ., Maringá, n.37, p. 173-194 jul./set. 2013.

STILLWELL, S.B. Searching for the evidence strategies to help you conduct a successful search. AJN., v. 110, n. 5, p. 41-7, 2010. 